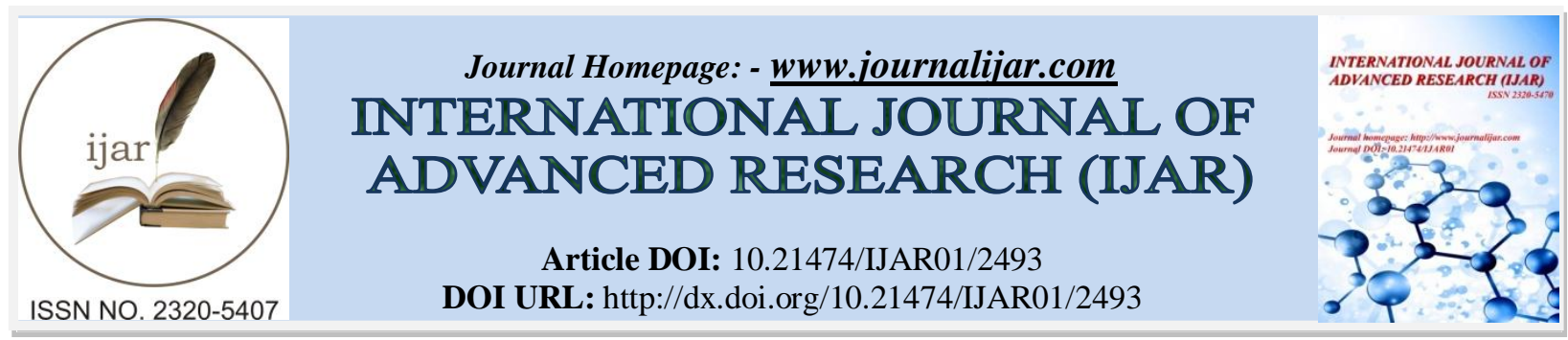

RESEARCH ARTICLE

\title{
HEAT FLUX MEASUREMENT AND NUMERICAL SIMULATION OF FLOWFIELD ON LENS DOUBLE CONE GEOMETRY.
}

Kavitha R.

Vimal Jyothi Engineering College, Kannur, Kerala, India.

\section{Manuscript Info}

Manuscript History

Received: 25 October 2016

Final Accepted: 23 November 2016

Published: December 2016

\section{Abstract}

This paper presents the numerical simulations of flow field over a typical $25^{\circ}-55^{\circ}$ sharp tipped axisymmetric double cone at different flow conditions using ANSYS Fluent. Detailed surface heat flux and pressure measurements over the model have been made with welldefined boundary conditions. Flow features such as shock interactions, location of the sonic line are captured very well in the present simulations. The simulation distribution shows excellent match with the experimental results taken from the database of Calspan University at Buffalo Research Centre (CUBRC).

Copy Right, IJAR, 2016,. All rights reserved.

\section{Introduction:-}

Hypersonic flow separation over bodies continues to intrigue researchers, since considerable differences are observed between experiments and CFD. The interaction between a shock wave and boundary layer often results in local regions of separated flows. Upstream facing corner formed by a deflected control surface on a boundary layer in hypersonic re-entry vehicle, internally generated shock wave impinging on a boundary layer in hypersonic air breathing propulsion system and separation bubble induced by control surface deflection are some of the typical scenarios where precise knowledge of separated flow features are essential.

A double cone model is a useful configuration for studying the 3-D separated flow features under severe adverse pressure gradient. Depending on the first and second apex angles the flow field around the double cone will comprise of several classical viscous flow features such as shock wave/boundary layer interaction, recirculation zones etc.

As the scope and versatility of CFD methods have advanced the inevitable assumption has been made that sooner or later numerical predictions methods would replace wind tunnel tests for all flow problems. In honesty so far this has proved to be overly optimistic and, although spectacular improvements have been made with CFD, it appears that there are critically important flow phenomena that cannot be predicted very accurately and it is even been questioned if the governing physics can be captured correctly.

An over confidence in computer prediction methods appears to have been a serious error. It has unfortunately led to the demise of many experimental facilities, which are essential both to validate the computer solutions and to provide measured data that can be relied upon for many difficult and complex problems.

Errors in CFD predictions can arise from a variety of sources. At the most fundamental level, the appropriateness and validity of the underlying fluid dynamic formulation has to be questioned, as it may be inadequate to describe 
the flows correctly. Much more obviously, the numerical procedures used to solve the underlying equations may well be at fault. All depend on algorithms that are only accurate to a certain order, all depend on spatial discretisation for which a high-quality mesh, matched appropriately to flow details to be derived, and all depend on computer software and machines that may not be perfect. To a certain extend questions such as the degree to which a solution has converged or whether there is sufficient spatial resolution can be determined from internal evidence from running the code itself, but it is also important to compare the results with corresponding solutions of the same problem using alternative numerical techniques, computational meshes etc. from other researchers. Such a process is called verification and is principally aimed at exposing errors due to incorrect implementation of the codes or identifying differences between the various numerical techniques and levels of accuracy adopted. Verification cannot answer the questions as to whether a correct fluid dynamic formulation has been used or if the CFD method is intrinsically capable of capturing the physics and giving correct answers. This can only be done by comparing the computed results with carefully derived experimental data for matching boundary conditions and body geometry. To do this meaningfully high integrity measured data have to be available, of a quality such that the uncertainties in these are less than any disagreements with the CFD results. This comparison process is called validation and is far better done if the participating parties have no prior knowledge of the result of the other researchers, and especially the equivalent experimental results. If this is so, it is referred to as blind validation and the outcome is of greater value. There is a natural reluctance for CFD researchers to publish results that disagree with the experiment and vice-versa and hence failures in the correlation between measured and computed data are often not made public. There is always a temptation to rerun calculations or use alternative schemes until agreement is achieved before publishing and hence the true picture is often obscured. This most certainly is not to suggest that cheating is commonplace but there is obvious merit in setting up blind validation exercises in order to get a truer picture especially if a number of different laboratories are prepared to participate.

This paper will present the results of a blind validation exercise that has been organized around on the CUBDAT experimental database, which has been assembled by the CUBRC from tests performed in the LENS wind tunnel. This data base contains a very large volume of results covering a wide variety of flow conditions.

Most of the CFD codes currently in use are based on a Navier-Stokes formulation. It is generally accepted that these equations are intrinsically accurate and thus any failure experienced in obtaining perfect answers has to be attributable to errors in the choice or implementation of the numerical technique used to solve them as opposed to any shortcoming in the equations themselves. There are many numerical schemes available to solve these equations, which range widely in precision and computational efficiency- generally the more accurate being the most demanding on computer resources and the most difficult and the slowest with which to obtain stable converged solutions. The accuracy of a solution also depends on the fineness and quality of the computational mesh used and to how well it relates to the structure of the flow being computed. This is particularly true for flows such as those being considered in this exercise, where complicated shock wave and shear layer features are present. Adapting meshing techniques have been developed to deal with this difficulty. One of the objectives of the present validation exercise is thus to identify what level of sophistication in numerical technique and mesh structure is required to obtain adequately accurate solutions for each flow example if indeed such solutions can be obtained.

The hypersonic flow over double cone geometry is an interesting computational fluid dynamics (CFD) case because it produces many of the complex phenomena, such as shock interactions, triple points, and recirculation zones that take place in flows past hypersonic vehicles. These vehicles will likely have regions of separated flow, for example, near control surfaces and behind flame holders. Additionally, shock-shock interactions and shock impinging on the vehicle surface cause high localized aero thermal loads.

\section{Methodology:-}

A systematic study of the effects of the numerics on the simulation of a steady hypersonic flow past a sharp double cone configuration is challenging to compute. The simulation of flow hypersonic CFD plays an important role in the development of hypersonic vehicle. Shock/shock and shock/boundary layer interactions can seriously degrade the performance of a hypersonic vehicle. The complexity and the design implications of these phenomena require their quantitative assessment. The precise calculation of supersonic and hypersonic flows put conflicting demands on the formulation of inviscid numerical flux functions. A high speed numerical scheme has to possess enough dissipation to capture strong shocks without developing overshoots and oscillations in the vicinity of the discontinuity and the scheme must also possess numerical dissipation that is much smaller than the physical viscosity to accurately compute boundary layers. Investigation shows that simulation accuracy is directly depended 
on the grid algorithm. However, several conclusions in previous research are not entirely consistent consequently making the development of an accurate simulation system of hypersonic CFD difficult. Experimental data on hypersonic sharp double-cone flow are used in the aim to provide a basis for hypersonic simulation CFD software.

\section{Computational procedure:-}

The axisymmetric flow past the sharp double cone is governed by the compressible, time dependent Navier-Stokes equations

$$
\int ð U / \partial t d V+\int F . n d S=\int W d V
$$

Where $U=(\rho, \rho u, \rho v, \rho e)^{\prime}$ is the vector of conserved variables, $F$ the vector of convective and dissipative fluxes, and $\mathrm{W}$ the vector of axisymmetric source terms. Because the gas is nonreactive, the transport coefficients are computed according to the usual laws: The fluid viscosity is temperature dependent and is given by Sutherland's law,

$$
\mu=1.458^{*} 10^{-6} \mathrm{~T}^{3 / 2}(\mathrm{~T}+110.4) \mathrm{kg} / \mathrm{m} \mathrm{s}
$$

Thermal Conductivity is given by

$$
\lambda=\mu \mathrm{cp} / \mathrm{Pr}
$$

Where cp is the specific heat at constant pressure and the Prandtl number $\operatorname{Pr}=0.72$.

The equations are discretised in space according to the finite volume method, where the fluxes at the interfaces of each cell are evaluated with appropriate Riemann solvers. The careful time convergence study on this test case and showed that the solution does not converge until at least 100 characteristic flow times have been computed. This relatively long timescale allows the separation zone to become fully established. They define the characteristic time to be the time required to travel the length 1 of the double cone model at the freestream velocity. Our computations are consistent with their findings, and we simulate our flow to a minimum of 150 flow times.

To obtain the grid-converged solution, we use grids where the smallest dimension $\Delta \mathrm{y}$ min is of the order of $0.1 \mu \mathrm{m}$. For stability reasons, the explicit computational time allowed, in which the Courant-Friedrichs-Lewy (CFL) number plays a major role. Then, to obtain the steady-state solution with an explicit time integration method, we would need $\mathrm{N}_{\text {expl }}$ iterations, with $\mathrm{N}_{\text {expl }}=100 \tau_{\text {char }} / \tau_{\text {expl }}$. With the characteristic size being about $0.2 \mathrm{~m}, \mathrm{~N}_{\text {expl }}$ is at least $2 \times 108$. It would be impossible to run an explicit Navier-Stokes code for this huge number of time steps, and we must use an approach that converges more rapidly than an explicit method. In the present work, we use an implicit time integration approach.

\section{Grid requirements:-}

The solution of the Navier-Stokes equations is an extremely expensive endeavor. The cost stems from the need to cluster cells near the body in order to capture the important viscous phenomena. This clustering has two consequences. Firstly we need more cells to discretise a given region. Secondly the small normal spacing increases the stiffness of the extent that expensive implicit schemes are necessary. Needless to say, a grid that gives the necessary resolution with the minimum number of cells in a highly desirable goal. Therefore appropriate attention is being given to the construction of a good grid.

To obtain the optimal resolution for a given number of grid points, it is necessary to group points into regions where the state vector is changing, most rapidly. Since this is a viscous calculation, we know that the boundary layer is one such region. Within the boundary layer, changes in the body normal direction dominate; therefore fine spacing must be maintained in this direction. Therefore it is important to impose cell face orthogonality in the boundary layer in order to maintain solution accuracy.

\section{Implementation of the boundary conditions:-}

For the numerical simulation, Boundary conditions are required on the Double cone surface, at the wall, farfield and symmetry boundaries.

\section{Overview of flow solvers:-}

Ansys Fluent allows one of the two numerical methods:

- Pressure-based solver

- Density-based solver 
The pressure based approach was developed for low-speed compressible, while the density based approach was mainly used for high speed compressible flows.

In both methods the velocity field is obtained from the momentum equations. In the density-based approach, the continuity equation is used to obtain the density field while the pressure filed is determined from the equation of state.

\section{Density-Based Solver:-}

The density based solver solves the governing equations of continuity, momentum and energy and species transport simultaneously. Because the governing equations are non-linear, several iterations of the solution loop must be performed before a converged solution is obtained. Each iteration consists of the steps outlined below:

1. Update the fluid properties based on the current solution.

2. Solve the continuity, momentum, and energy and species equations simultaneously.

3. Where appropriate, solve equations for scalars such as turbulence and radiation using the previously updated values of the other variables.

4. When interphase coupling is to be included, update the source terms in the appropriate continues phase equations with a discrete phase trajectory calculation.

5. Check for convergence of the equation set.

6. These steps are continued until the convergence criteria are met.

The manner in which the governing equations are linearised may take an "implicit" or "explicit" form with respect to the dependant variable of interest.

Implicit: For a given variable, the unknown value in each cell is computed using a relation that includes both existing and unknown values from neighboring cells. Therefore each unknown will appear in more than one equation in the system, and these equations must be solved simultaneously to give the unknown quantities.

\section{Modelling:-}

The measurements made in a number of experimental programs designed to obtain fundamental measurements for code validation in regions of shock wave/laminar boundary layer interaction and shock/shock interaction flows at Mach numbers between 10 and 14 for a range of Reynolds numbers. Detailed measurements of heat transfer and pressure made on a axisymmetric double cone model and free stream test conditions selected for these studies generated flows in which the regions up and downstream of the separated regions were attached, and the pressure and heat transfer in these regions could be easily predicted. The studies were made over a large Reynolds number range to ensure that the flows selected for comparison with laminar numerical solutions remained fully laminar downstream of the interaction region.

\section{Model configuration:-}

The configuration used in this study is a sharp double cone which has a half angle of $25^{\circ}$ of the first and $55^{\circ}$ of the second cone. The base radius of the model is $130.9243 \mathrm{~mm}$ and the overall length is $193.675 \mathrm{~mm}$. A sketch of the configuration is shown in the fig 3.1

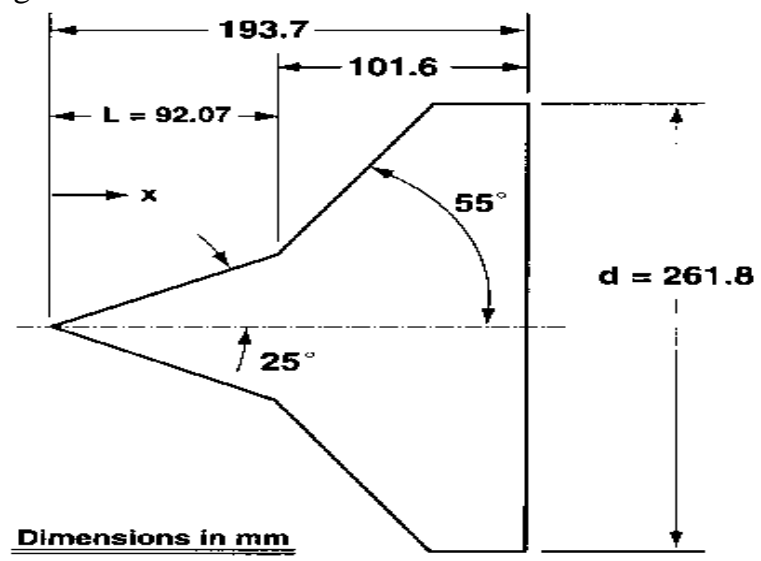

Figure 3.1:- Double Cone Model Dimension Diagram 
The configuration is axisymmetric and so avoids the experimental uncertainty that occur with supposedly "two dimensional" tests on, for example, flat plates and wedges, due to the finite span of the wind tunnel models.

Earlier tests on a $65^{\circ}$ aft cone proved to be unsatisfactory as the flow was not steady. However, with the lower cone angles of $55^{\circ}$ and $60^{\circ}$ steady flows were realized as witnessed by the time history from the gauges on these and from high speed Schlieren videos of the flow.

\section{Computational domain and grid:-}

Figure 3.2 shows the grid distribution in the domain and on the model surface. Computations are carried out on multi block structured grid using Fluent for half model to reduce the computational time. A total of 4 blocks are used to generate the grid over the model and the total number of elements is 11700 . The surface grid distribution is based on the level of resolution required for strategic flow physics. For example, attention is given near the surface of the model especially on the first and second cone. The first cell from the wall is of the order of $10^{-} 8 \mathrm{~m}$ with a succession ratio of 1.1 is provided to accurately predict the boundary layer flow and uniform transition from fine to coarse grid.

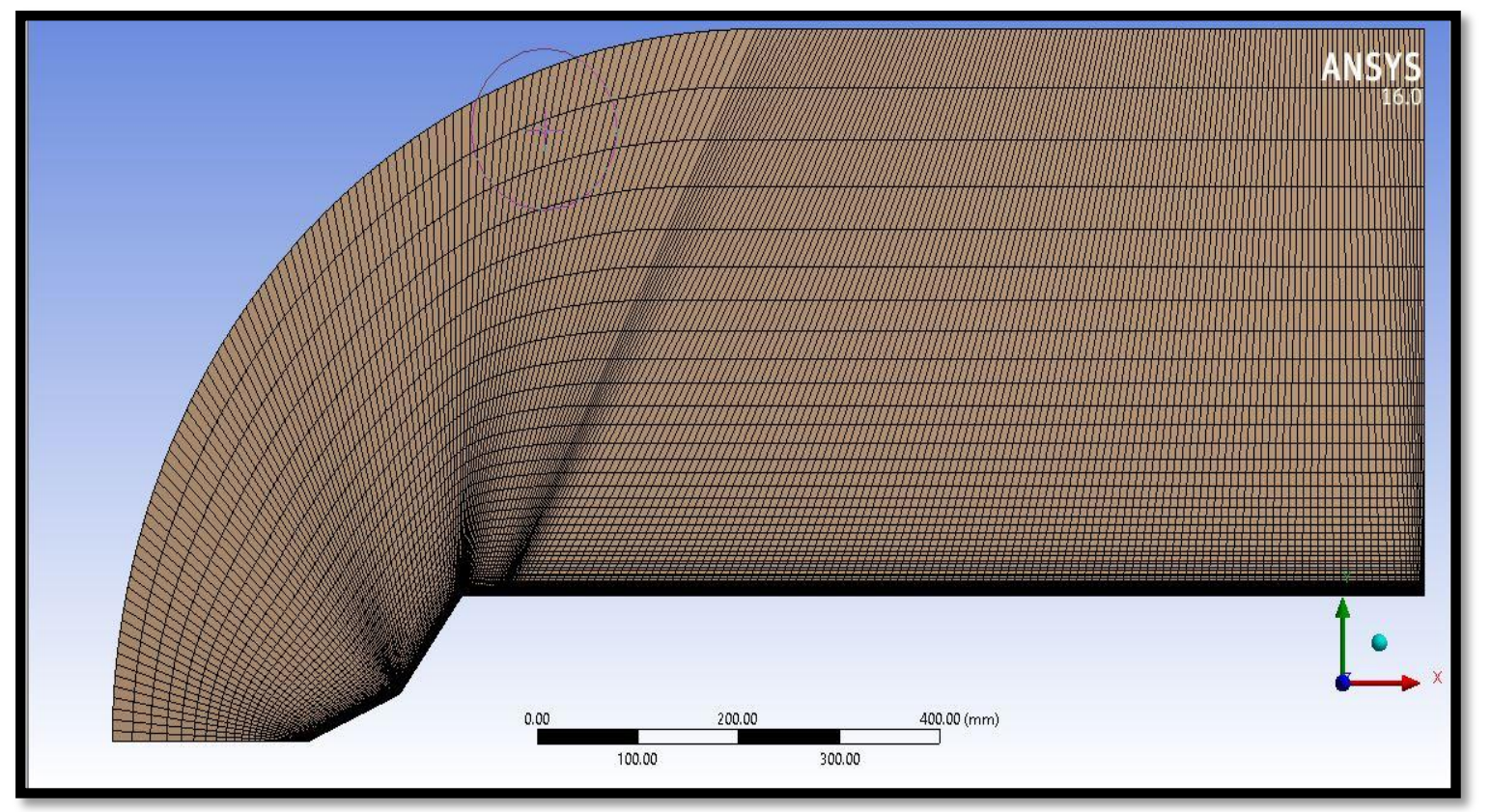

Figure 3.2:- View of computational domain and grid

The double cone flow has several critical flow features that require careful grid generation. First, at the tip of the first cone, the grid must be well resolved to capture the boundary layer that starts developing at this location. At the junction of the two cones, the grid lines must be smooth to not introduce artificial perturbation. The grid is highly stretched to the surface and that care has to be taken maintain grid regularity and smoothness as much as possible.

\section{Flow solver and solution procedure:-}

The simulations are carried out using an evaluation version of the commercial code ANSYS FLUENT. The flow is assumed to be ideal, fully laminar and the effect of molecular viscosity is negligible. A three coefficients Sutherland's formula is used to compute the viscosity. The compressible flow equations are solved using implicit, density based solver. An implicit second order upwinding scheme is used for spatial discretisation. Boundary layer is resolved by enhanced wall treatment (solve to wall). The flux splitting scheme for convective terms are evaluated by Roe flux-difference splitting (Roe-FDS) scheme.

\section{Boundary conditions and flow parameters;-}

The boundary condition applied to the entire computational domain is pressure farfield where freestream Mach number, freestream static pressure, and the freestream static temperature are prescribed. The double cone surfaces are modeled with no slip boundaries. Wall surface temperature is assumed constant at $300 \mathrm{~K}$. 
Table 3.1:- Flow Conditions.

\begin{tabular}{|l|l|l|l|l|l|l|l|}
\hline RUN & $\begin{array}{l}\text { Total } \\
\text { Enthalpy } \\
(\mathrm{MJ} / \mathrm{kg})\end{array}$ & $\begin{array}{l}\text { Mach } \\
\text { Number }\end{array}$ & $\begin{array}{l}\text { Pitot } \\
\text { Pressure }(\mathrm{kPa})\end{array}$ & $\begin{array}{l}\text { Unit Reynolds } \\
\text { Number/10 } \\
(1 / \mathrm{m})\end{array}$ & $\begin{array}{l}\text { Velocity } \\
(\mathrm{km} / \mathrm{s})\end{array}$ & $\begin{array}{l}\text { Density } \\
\left(\mathrm{g} / \mathrm{m}^{3}\right)\end{array}$ & $\begin{array}{l}\text { Temperature } \\
(\mathrm{K})\end{array}$ \\
\hline 01 & 5.44 & 12.2 & 5.1 & 0.14 & 3.246 & 0.499 & 175 \\
\hline 02 & 9.65 & 10.9 & 17.5 & 0.19 & 4.303 & 0.984 & 389 \\
\hline 03 & 18.70 & 13.23 & 18.0 & 0.11 & 6.028 & 0.510 & 521 \\
\hline 04 & 21.77 & 12.82 & 39.5 & 0.20 & 6.497 & 0.964 & 652 \\
\hline 05 & 18.51 & 13.14 & 36.8 & 0.23 & 5.996 & 1.057 & 523 \\
\hline 06 & 15.23 & 11.46 & 59.0 & 0.39 & 5.466 & 2.045 & 573 \\
\hline
\end{tabular}

For this flow, the boundary conditions are supersonic inflow, symmetry on the cone axis, and a no-slip isothermal surface on the cone. Computed velocities remained parallel to the boundary.

\section{Convergence study:-}

The double cone flow fields are initialized with freestream conditions, and the solution is integrated in time until it reaches a steady state. To assess solution convergence, residual equations are monitored during iterations. It is assumed that the solution is converged when the residuals drop more than the three orders of magnitude. Fig shows the convergence log for Navier Stokes equation for nominal grid where the residuals drop is found to be more than three orders of magnitude. It can be observed that the solutions are assumed to be converged after 30000 iterations.

Initially the solutions are first order accuracy. Once the solution reaches steady state, second order is turned on. This can be seen that up to 20000 iterations, the solutions are steady except some oscillations are seen in the initial stage. Once the second order is initiated after first order is well converged after iterations, a drop is seen flowed by initial oscillations. The solution becomes steady after iterations. However the computations are carried out for enough time to see the solutions are completely constant.

The use of an implicit method allows us to run at time steps as large as possible to obtain results with the smallest number of iterations and, therefore, within the shortest computational time. Most computations used time steps of the order of $1 / 100$ th $-1 / 1000$ th of the characteristic flow time. Thus given that the flowfield requires at least 100 flow times to establish, the solution should require roughly $10^{3}-10^{4}$ time steps to reach convergence. However, even if the implicit code can be run with very large time steps, it is not always beneficial to use the largest possible time steps. If the time step is too large, the computation may not converge as quickly as with a smaller time step; the residual may oscillate without decreasing, even if the flow has already attained the steady state. If the time step is even larger, the shock position keeps oscillating, and the flow never reaches a steady state. Thus, there is an optimal value of the maximum time step for each method.

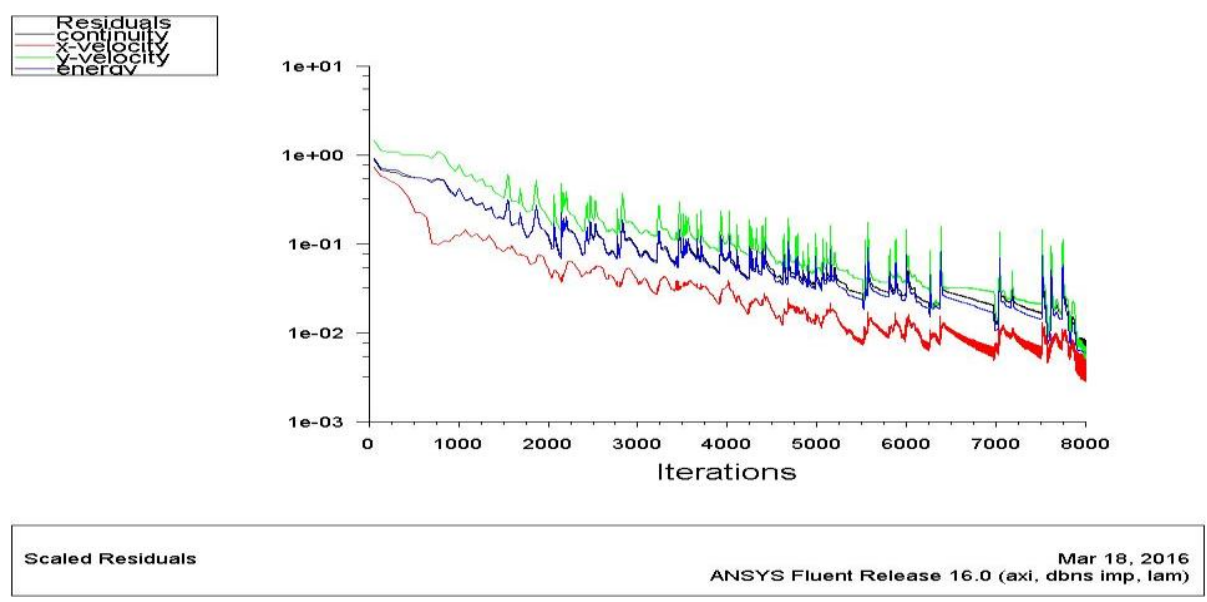

(a) 


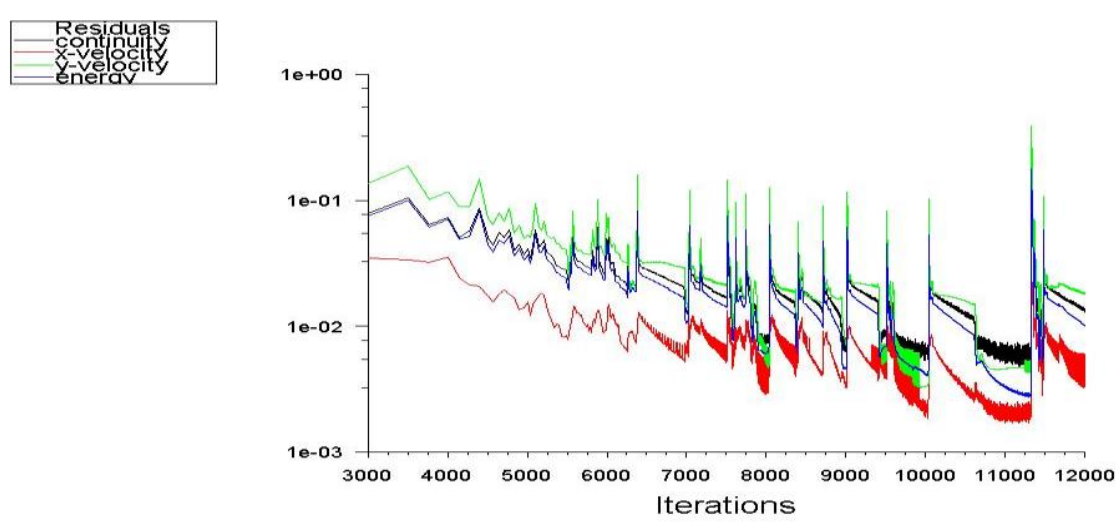

Scaled Residuals $\quad$ Mar 18,2016
ANSYS Fluent Release 16.0 (axi, dbns imp, lam)

(b)

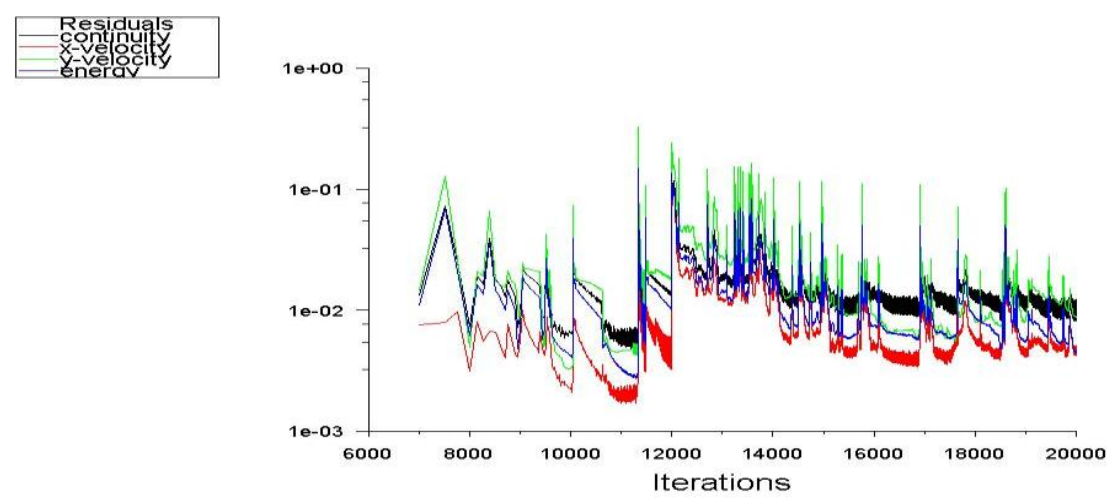

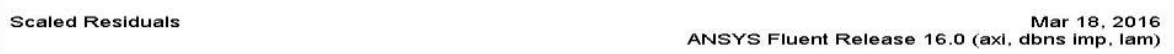

(c)

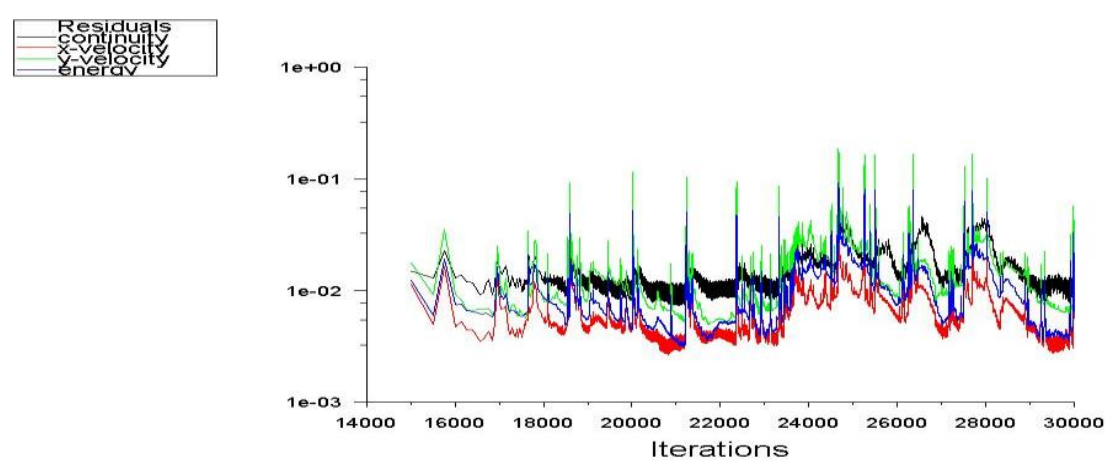

Scaled Residuals ANSYS Fluent Release 16.0 (axi, dbns imp. lam)

d)

Figure 3.3:- Grid Convergence History (a-d)

\section{Results and Discussions:-}

The model created is subjected to analysis with the pre mentioned boundary conditions and is compared with the experimental value. The experimental measurement of wall pressure and heat flux has been taken from the database of the Calspan University at Buffalo Research Center (CUBRC) to confirm the simulation accuracy of numerical simulation software for laminar flow hypersonic separation flow. 
Experimental heat flux and wall pressure values:-

The experimental cases selected as benchmark are shown in table 4.1 and 4.2

Table 4.1:- Double cone surface heat flux $\left(\mathrm{W} / \mathrm{cm}^{2}\right)$ - Experimental

\begin{tabular}{|c|c|c|c|c|c|c|}
\hline $\mathrm{X}(\mathrm{cm})$ & Run01 & Run02 & Run03 & Run04 & Run05 & Run06 \\
\hline 2.16 & 51.196 & 168.316 & 362.124 & & & \\
\hline 2.41 & 46.853 & 153.317 & 331.493 & 588.848 & 495.148 & 536.693 \\
\hline 2.62 & 44.532 & 146.063 & 316.521 & 571.46 & 474.099 & 509.865 \\
\hline 3.38 & 40.816 & 132.972 & 291.029 & 522.022 & 431.662 & 467.249 \\
\hline 3.61 & 38.49 & 124.679 & 277.602 & 495.376 & 414.818 & 451.203 \\
\hline 3.84 & 38.581 & 125.056 & 279.253 & 498.383 & 414.578 & 449.29 \\
\hline 4.09 & 38.813 & 121.015 & 285.561 & 494.048 & 405.408 & 450.482 \\
\hline 4.32 & 36.475 & 117.078 & 265.325 & 474.252 & 389.197 & 420.115 \\
\hline 4.52 & 37.589 & 112.613 & 276.38 & 486.878 & 388.669 & 424.938 \\
\hline 4.78 & 36.08 & & & & & \\
\hline 5 & 33.72 & 109.967 & 249.985 & 434.11 & 362.781 & 395.612 \\
\hline 5.23 & 32.823 & 107.309 & 242.506 & 428.111 & 354.23 & 383.984 \\
\hline 5.46 & 32.283 & 105.908 & 239.663 & 425.491 & 348.368 & 372.821 \\
\hline 5.69 & 30.835 & 100.39 & 228.293 & 408.98 & 332.291 & 361.877 \\
\hline 6.27 & 30.761 & 99.172 & 228.929 & 409.136 & 330.011 & 349.275 \\
\hline 6.5 & 29.572 & 92.755 & 221.229 & 389.591 & 323.821 & 198.706 \\
\hline 6.73 & 28.495 & 52.875 & 215.33 & 380.559 & 316.191 & 73.674 \\
\hline 6.93 & 12.236 & 39.964 & & 392.644 & 322.431 & 58.479 \\
\hline 7.19 & 8.684 & 26.92 & 209.569 & 369.947 & 153.096 & 61.213 \\
\hline 7.42 & & & & 337.128 & 67.388 & 65.413 \\
\hline 7.65 & 3.83 & 26.215 & 188.419 & 108.156 & 68.547 & 63.685 \\
\hline 7.87 & 2.954 & 27.648 & 153.304 & 80.504 & 65.664 & 71.119 \\
\hline 8.1 & 2.618 & 28.141 & 109.376 & 78.173 & 66.454 & 73.393 \\
\hline 8.33 & 2.787 & 31.849 & 39.844 & 95.136 & 70.839 & 82.252 \\
\hline 8.56 & 1.789 & 32.329 & 31.146 & 97.536 & 75.548 & 92.757 \\
\hline 8.79 & 1.791 & 31.735 & 30.887 & 105.039 & 79.796 & 113.098 \\
\hline 9.37 & 4.779 & 23.309 & 126.05 & 138.185 & 59.233 & \\
\hline 9.5 & 11.135 & 59.128 & 306.838 & 579.059 & & \\
\hline 9.73 & 17.883 & 124.027 & 538.118 & & & \\
\hline 10.01 & 20.549 & 140.942 & & 774.636 & 702.54 & \\
\hline 10.24 & 30.87 & 235.485 & 120.959 & 619.133 & 667.766 & 1020.196 \\
\hline 10.46 & 57.914 & 378.001 & 290.293 & 515.839 & 474.119 & 787.03 \\
\hline 10.74 & 91.302 & 175.659 & 238.57 & 492.819 & 440.96 & 512.788 \\
\hline 10.97 & 60.383 & 104.427 & 223.161 & 626.459 & 421.565 & 427.633 \\
\hline 11.18 & 45.483 & 131.669 & 257.479 & 636.379 & 462.976 & 374.67 \\
\hline 11.48 & 39.657 & 129.977 & 274.194 & 536.608 & 465.281 & 392.091 \\
\hline 11.68 & & 131.079 & 243.123 & 508.281 & & 374.881 \\
\hline 11.91 & 34.596 & 136.206 & 242.823 & 657.33 & 522.198 & 491.163 \\
\hline 12.22 & 40.964 & 171.526 & 303.035 & 619.271 & 522.24 & 505.883 \\
\hline 12.42 & 43.676 & 166.381 & 296.296 & 590.788 & 545.472 & 560.019 \\
\hline 12.65 & 45.436 & 169.611 & 292.369 & 523.199 & 490.982 & 471.82 \\
\hline 12.93 & 39.804 & 158.54 & 270.208 & 531.024 & 470.009 & 490.403 \\
\hline 13.16 & 37.365 & 148.636 & 262.302 & 479.42 & & 415.928 \\
\hline 13.36 & 35.722 & 147.366 & 251.759 & 500.597 & 383.308 & 467.671 \\
\hline 13.67 & 35.291 & 141.354 & 249.865 & 469.907 & & \\
\hline 13.87 & 34.847 & 140.776 & 253.868 & 507.115 & & \\
\hline 14.1 & 35.098 & 137.46 & & 520.552 & 414.183 & 437.754 \\
\hline 14.4 & 35.814 & 135.449 & & 540.741 & 432 & 434.076 \\
\hline 14.61 & 32.844 & 121.128 & & 559.288 & & \\
\hline 14.83 & 32.685 & 125.976 & & & & \\
\hline
\end{tabular}


Table 4.2:- Double Cone Surface Pressure $(\mathrm{kPa})$-Experimental3.

\begin{tabular}{|c|c|c|c|c|c|c|}
\hline $\mathrm{X}(\mathrm{cm})$ & Run01 & Run02 & Run03 & Run04 & Run05 & Run06 \\
\hline 4.04 & 1.048 & & & 8.685 & 7.486 & 11.843 \\
\hline 4.72 & & & & 7.62 & 7.185 & 11.75 \\
\hline 5.41 & 1.055 & 3.691 & 3.299 & 7.439 & 6.815 & 11.442 \\
\hline 6.1 & 1.075 & 3.248 & 4.107 & 8.2 & 7.133 & 11.32 \\
\hline 6.76 & 2.16 & 3.793 & 3.648 & 7.263 & 6.59 & 16.626 \\
\hline 7.49 & 2.25 & 7.608 & 2.964 & 10.455 & 10.24 & 22.526 \\
\hline 8.15 & 2.342 & 7.915 & 7.341 & 16.035 & 14.641 & 22.757 \\
\hline 8.86 & 2.35 & 8.021 & 8.612 & 16.455 & 15.033 & 24.329 \\
\hline 9.42 & & 9.76 & 12.108 & 24.62 & 25.522 & 28.79 \\
\hline 9.63 & 2.347 & 13.303 & 7.24 & 56.05 & 47.063 & 39.607 \\
\hline 10.06 & 2.582 & 26.491 & 26.055 & 54.312 & 60.007 & 95.866 \\
\hline 10.26 & 3.185 & 32.483 & 21.59 & 34.083 & 39.615 & 87.053 \\
\hline 10.49 & 3.804 & 25.593 & 11.288 & 24.157 & & 74.397 \\
\hline 10.69 & 4.345 & 13.831 & 9.441 & 21.805 & 23.67 & 48.616 \\
\hline 10.92 & 6.696 & 8.478 & 10.665 & 23.409 & 22.238 & 32.509 \\
\hline 11.15 & 5.922 & 8.444 & 11.685 & 26.317 & 23.784 & 38.067 \\
\hline 11.35 & 5.108 & 10.03 & 12.511 & 29.954 & 26.41 & 39.301 \\
\hline 11.58 & 4.558 & 12.232 & 12.819 & 30.714 & 28.483 & 40.876 \\
\hline 11.81 & 3.917 & 12.066 & 13.048 & 28.669 & 26.423 & 37.128 \\
\hline 12.01 & 3.663 & 13.3 & 13.28 & 27.966 & 26.072 & 41.341 \\
\hline 12.67 & 2.863 & 12.745 & 12.956 & 26.567 & 25.735 & 36.789 \\
\hline 13.34 & 3.432 & 12.974 & & & & \\
\hline 13.97 & 3.273 & 12.296 & 12.467 & 27.446 & 25.822 & 39.353 \\
\hline 14.63 & 2.91 & 11.814 & 12.561 & 27.647 & 25.614 & 37.491 \\
\hline
\end{tabular}

Theoretical heat flux and wall pressure values:-

A list of the computational values corresponds to the experimental test conditions have been received is shown in the table 6.3 and 6.4

Table 4.3:- Double cone surfaceheat flux $\left(\mathrm{W} / \mathrm{cm}^{2}\right)-$ Computational.

\begin{tabular}{|l|l|l|l|l|l|l|}
\hline $\mathrm{X}(\mathrm{cm})$ & Run01 & Run02 & Run03 & Run04 & Run05 & Run06 \\
\hline 0.00 & 59.87 & 175.65 & 380.25 & 601.2 & 556.114 & 559.125 \\
\hline 1.42 & 44.04 & 155.98 & 371.15 & 570.26 & 500.23 & 525.21 \\
\hline 2.96 & 42.93 & 137.68 & 305.96 & 548.1 & 481.056 & 515.56 \\
\hline 3.74 & 38.50 & 122.31 & 281.056 & 495.2 & 417.2 & 449.25 \\
\hline 4.70 & 36.50 & 115.23 & 245.35 & 451.2 & 370.154 & 390.2 \\
\hline 5.53 & 28.36 & 110.1 & 235.01 & 410.77 & 348.75 & 338.001 \\
\hline 6.85 & 15.00 & 45.259 & 210.14 & 330.25 & 310.44 & 50.21 \\
\hline 7.78 & 2.90 & 33.96 & 112.88 & 77.55 & 66.45 & 68.225 \\
\hline 8.90 & 1.75 & 20.56 & 28.65 & 144 & 55.65 & 105.33 \\
\hline 9.44 & 86.00 & 300.2 & 158.336 & 580.14 & 510.457 & 239.2 \\
\hline 10.30 & 55.00 & 384.68 & 530.26 & 769.13 & 624.17 & 1009.124 \\
\hline 10.80 & 42.60 & 177.2 & 226.33 & 505.187 & 678.6 & 545.012 \\
\hline 11.56 & 36.25 & 125.48 & 255.01 & 653.22 & 509.51 & 358.2 \\
\hline 12.40 & 40.68 & 140.62 & 283 & 596.44 & 410.112 & 505.4 \\
\hline 13.00 & 32.65 & 142.35 & 247.85 & 498.15 & 458.2 & 457.11 \\
\hline 14.70 & 31.65 & 122.03 & 253.2 & 522.017 & 404.2 & 453.01 \\
\hline 15.45 & 31.20 & 121.95 & 254.15 & 539.22 & 433.022 & 451.005 \\
\hline
\end{tabular}


Table 4.4:- Double Cone Surface Pressure (kPa) - Computational.

\begin{tabular}{|l|l|l|l|l|l|l|}
\hline $\mathrm{X}(\mathrm{cm})$ & Run01 & Run02 & Run03 & Run04 & Run05 & Run06 \\
\hline 0.00 & 0.98 & 2.225 & 2.758 & 6.598 & 7.43 & 11.65 \\
\hline 1.42 & 0.98 & 2.274 & 2.884 & 6.9558 & 7.65 & 11.74 \\
\hline 2.96 & 1.02 & 2.312 & 2.935 & 7.15 & 7.77 & 11.91 \\
\hline 3.74 & 1.03 & 2.444 & 2.9945 & 7.65 & 7.51 & 12.05 \\
\hline 4.70 & 1.04 & 3.015 & 3.0115 & 8.456 & 7.95 & 12.36 \\
\hline 5.53 & 1.12 & 3.73 & 3.125 & 7.45 & 7.132 & 11.56 \\
\hline 6.85 & 2.21 & 3.793 & 3.556 & 8.1 & 8.21 & 18.52 \\
\hline 7.78 & 2.28 & 7.52 & 3.132 & 12.25 & 12.25 & 25.2 \\
\hline 8.90 & 2.32 & 8.21 & 7.52 & 30.558 & 19.1 & 33.2 \\
\hline 9.44 & 2.35 & 12.44 & 12.52 & 53.256 & 28.91 & 42.114 \\
\hline 10.30 & 4.13 & 25.4 & 23.95 & 22.581 & 57.225 & 91.4 \\
\hline 10.80 & 5.23 & 30.15 & 15.225 & 25.145 & 26.51 & 71.7 \\
\hline 11.56 & 4.56 & 15.25 & 11.84 & 28.2258 & 29.8 & 37.025 \\
\hline 12.40 & 3.25 & 13.75 & 12.54 & 27.14 & 25.021 & 40.25 \\
\hline 13.00 & 3.36 & 12.5 & 12.12 & 27.114 & 24.965 & 39.6 \\
\hline 14.70 & 2.88 & 12.301 & 12.05 & 26.21 & 23.224 & 38.52 \\
\hline 15.45 & 2.87 & 12.221 & 11.855 & 26.201 & 23.2 & 38.353 \\
\hline
\end{tabular}

The heat flux and surface pressure distribution is plotted for both experimental values and theoretical values for each Run.

X-AXIS DENOTES THE AXIAL POSITION IN (cm) AND Y-AXIS DENOTES THE HEAT FLUX DISTRIBUTION $\left(\mathrm{W} / \mathrm{cm}^{2}\right)$

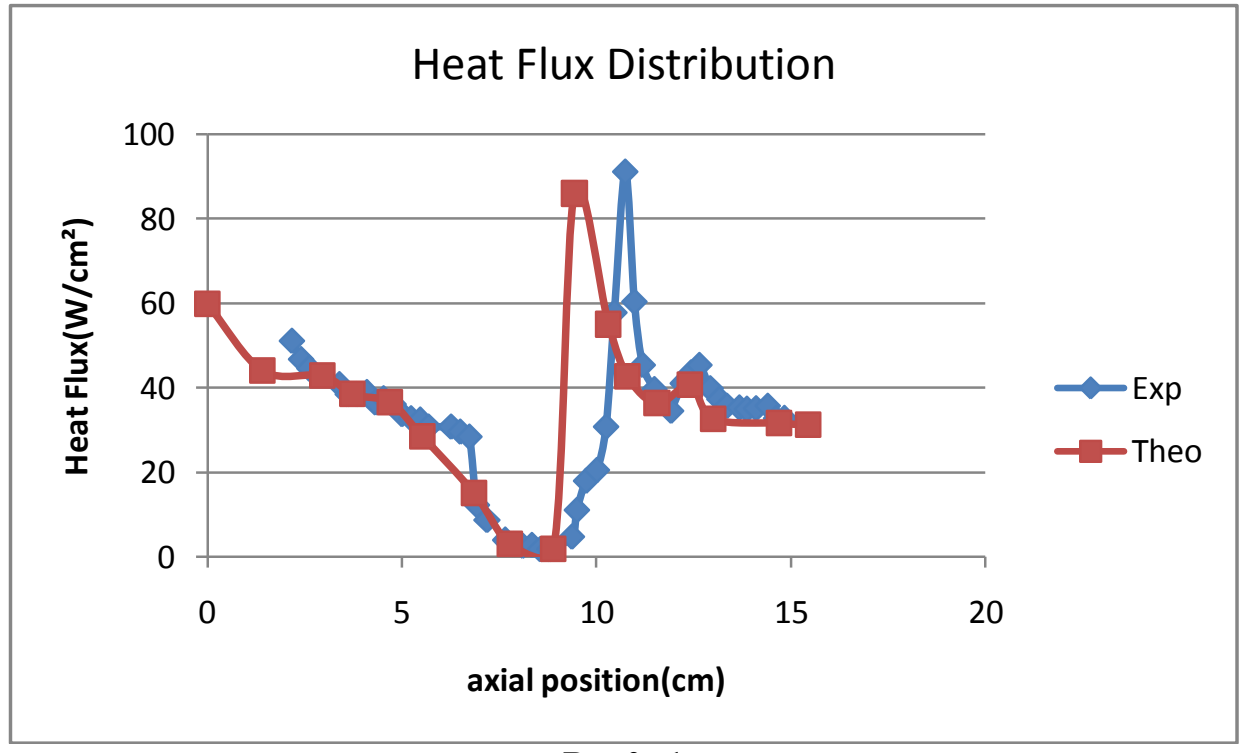

Run0:-1 


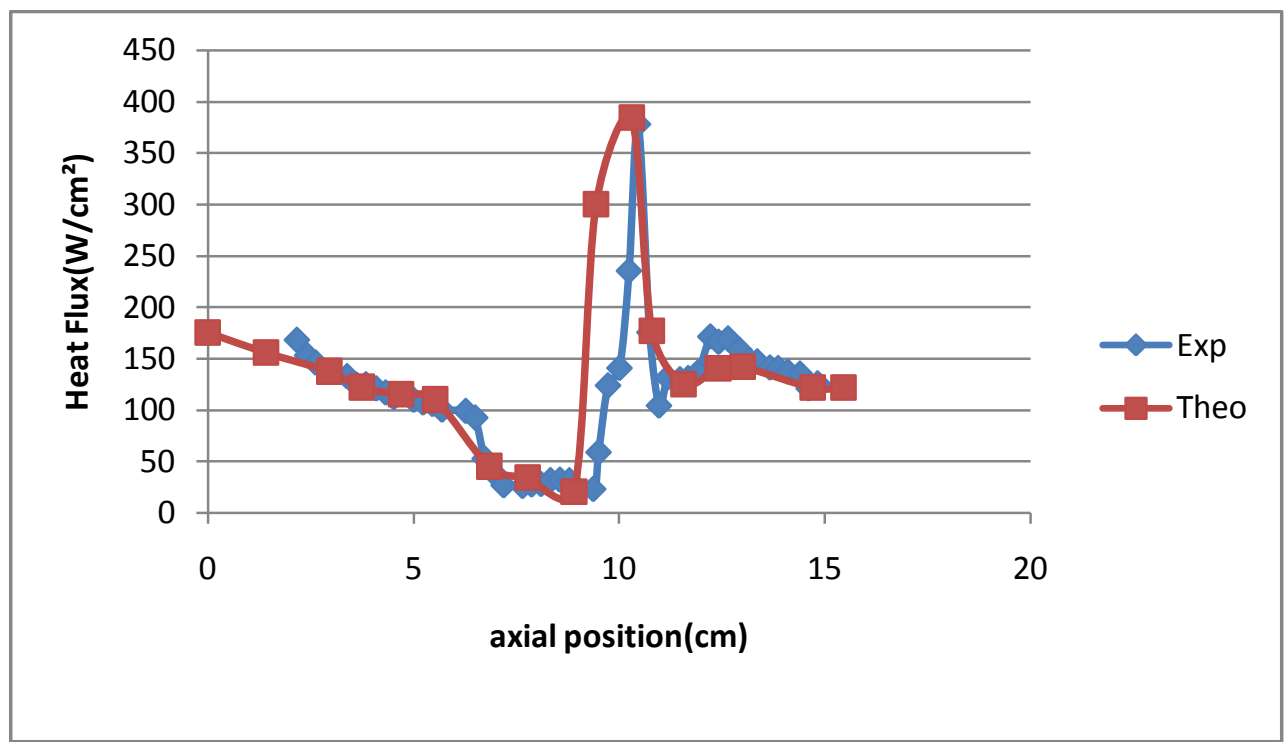

Run02:-

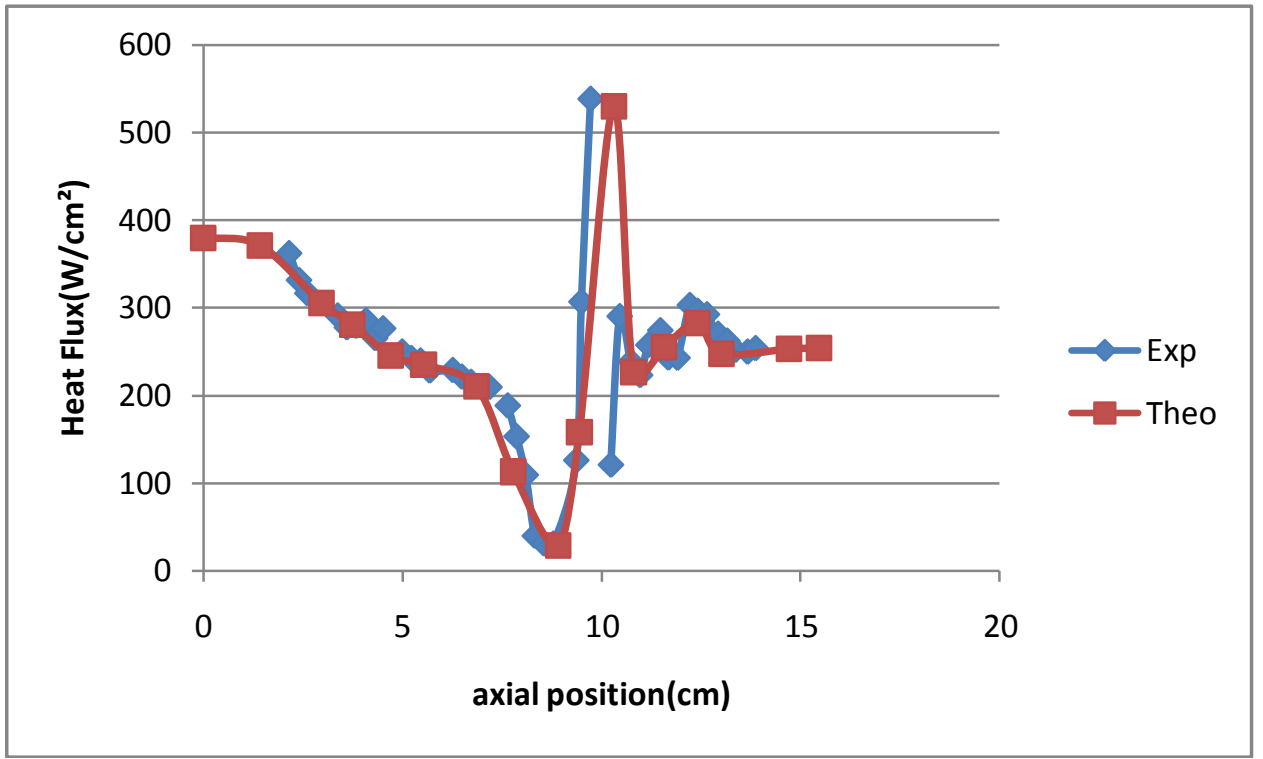

Run 03:- 


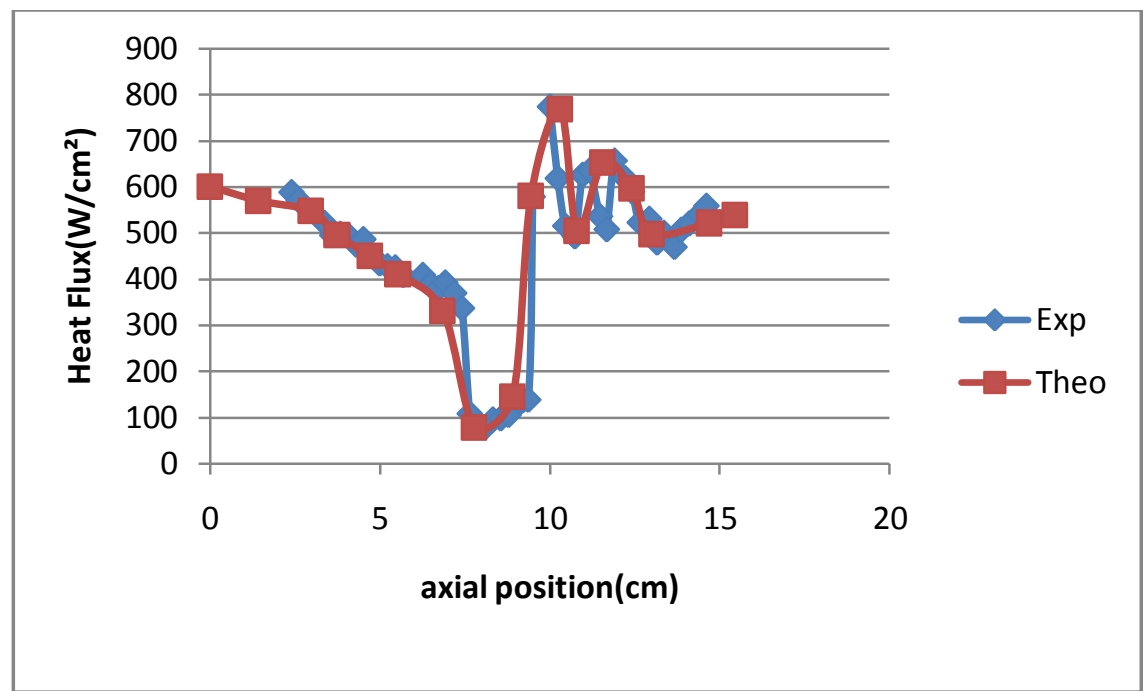

Run 04:-
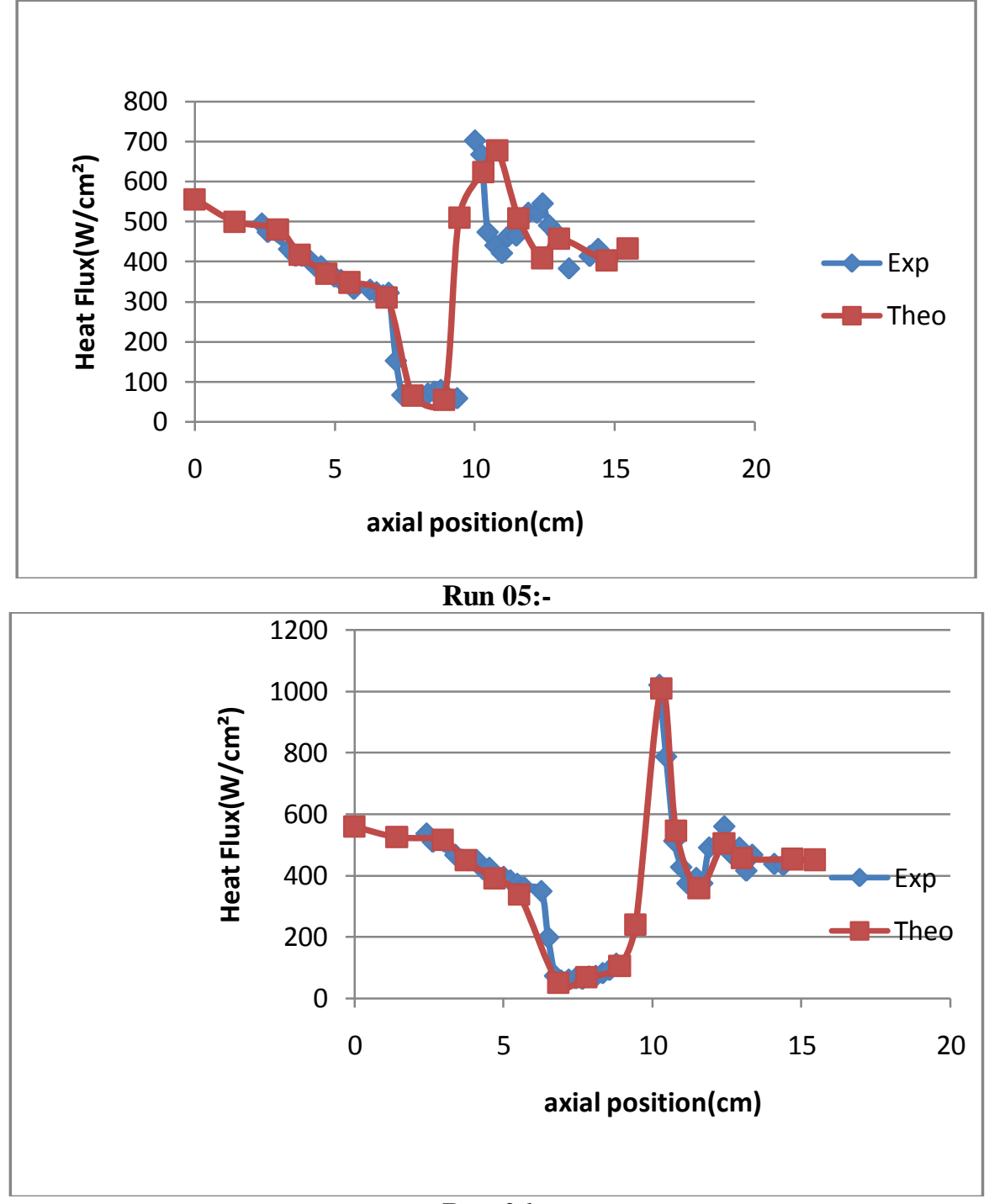

Run 06:-

Figure 4.1:- Surface Heat Flux Data Distribution for Double Cone Test Cases (RUN 01-RUN 06) 
X-AXIS DENOTES THE AXIAL POSITION IN (cm) AND Y-AXIS DENOTES THE SURFACE PRESSURE DISTRIBUTION $\left(\mathrm{W} / \mathrm{cm}^{2}\right)$

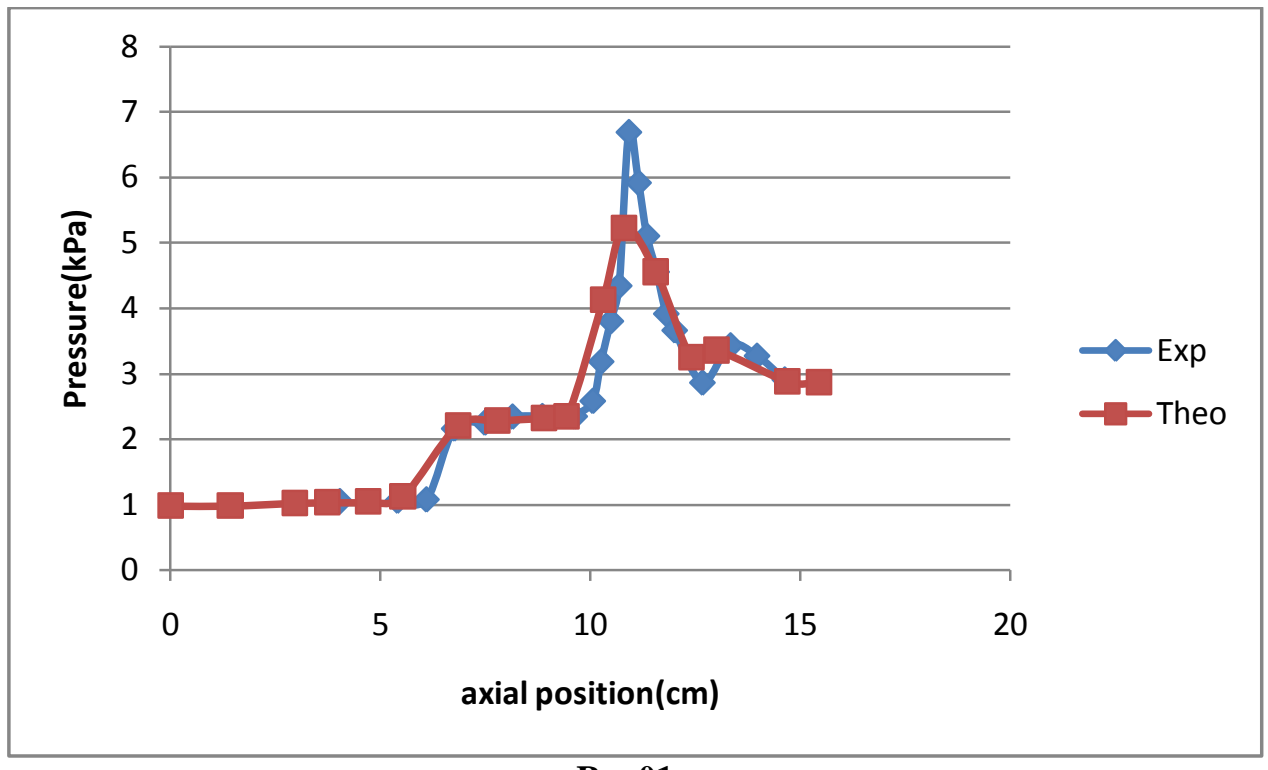

Run01:-

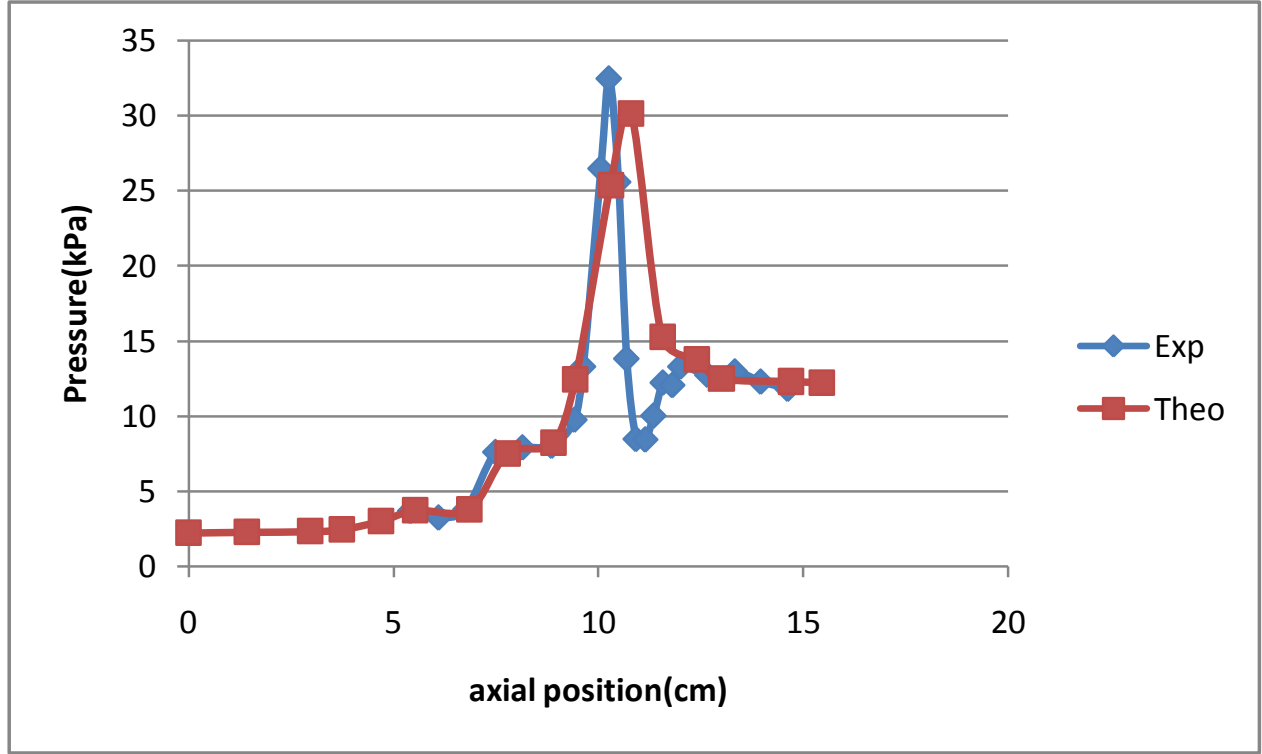

Run 02:- 


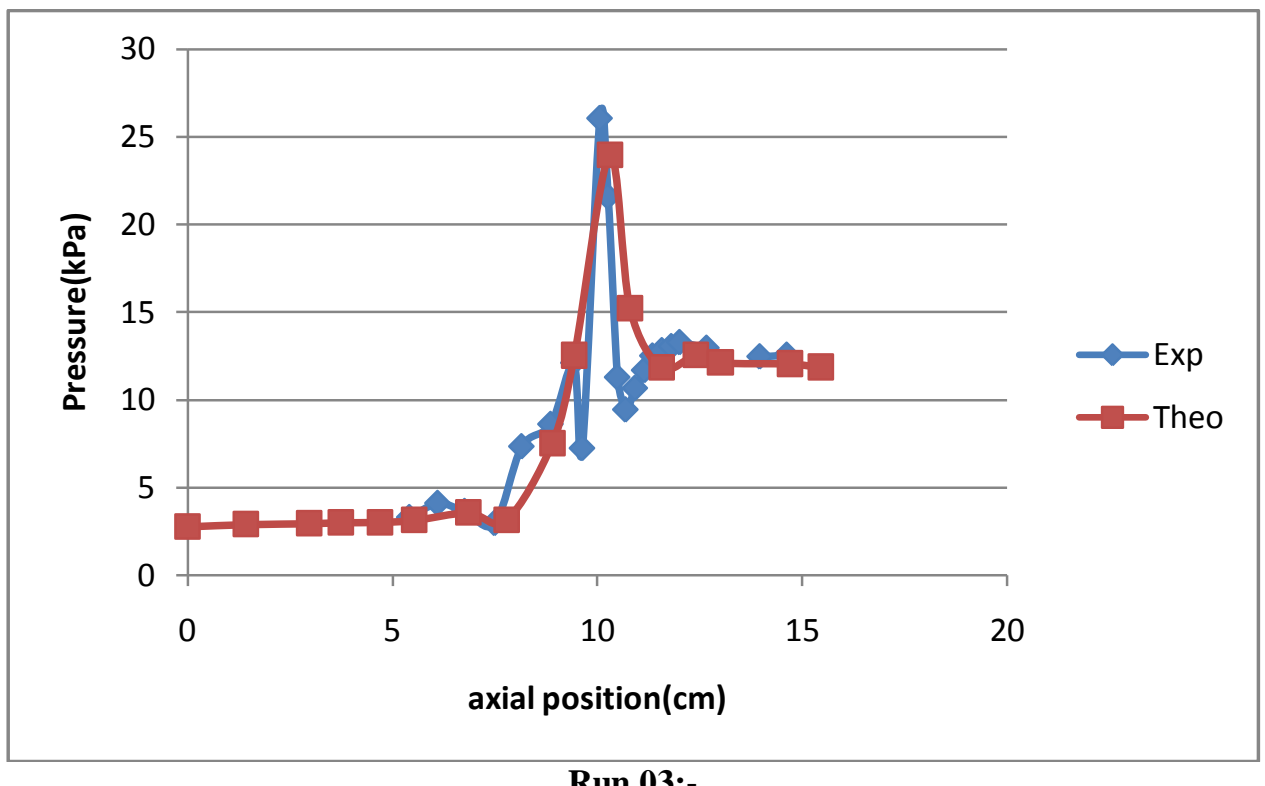

Run 03:-

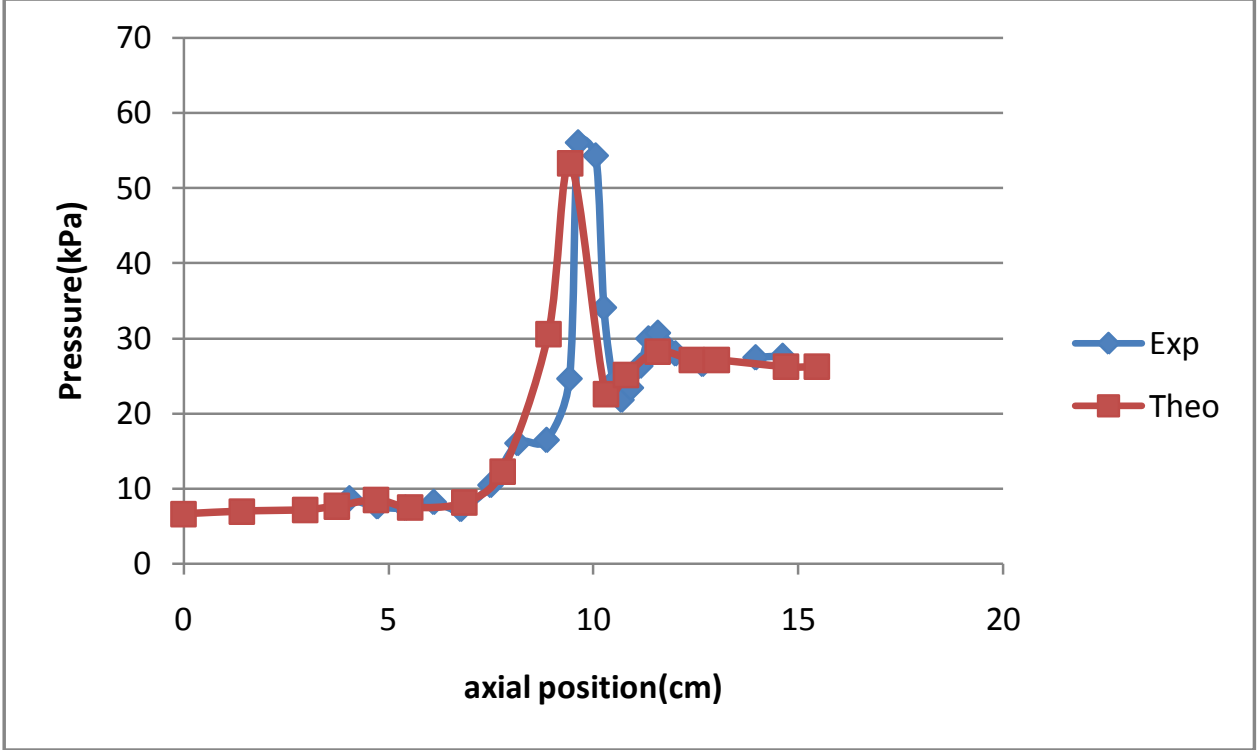

Run04:- 

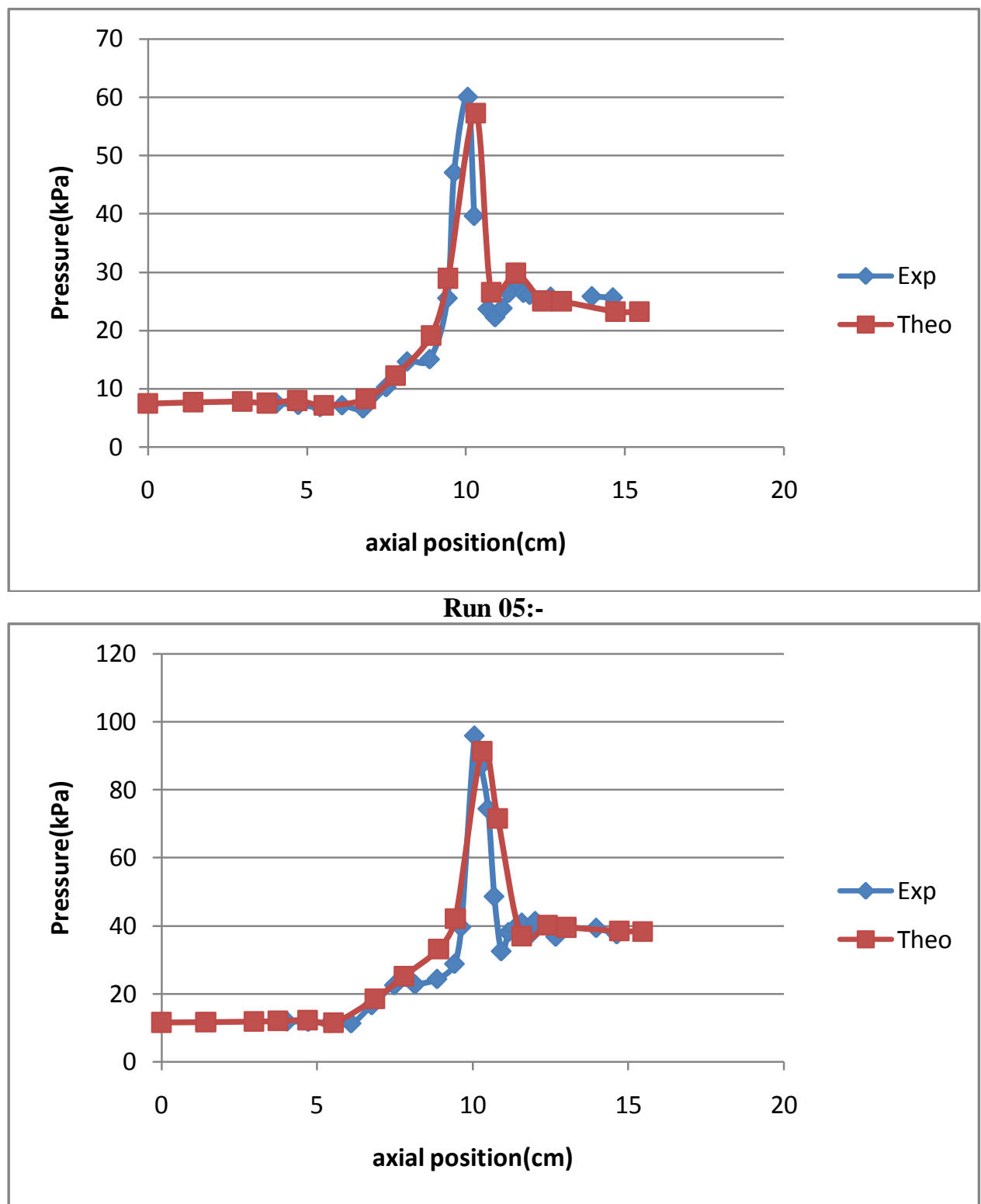

Run 06:-

Figure 4.2:- Surface Pressure Data Distribution for Double Cone Test Cases (RUN01-RUN06).

In general, consistency is observed between the surface pressre and surface heat flux- seperation and peak reattachment levels occur at about the same location within each run.

As the inducted shock wave interacted with the boundary layer, a high temperature and high pressure zone was formed. A high temperature leads to a high wall heat flux. 


\section{Flow features:-}

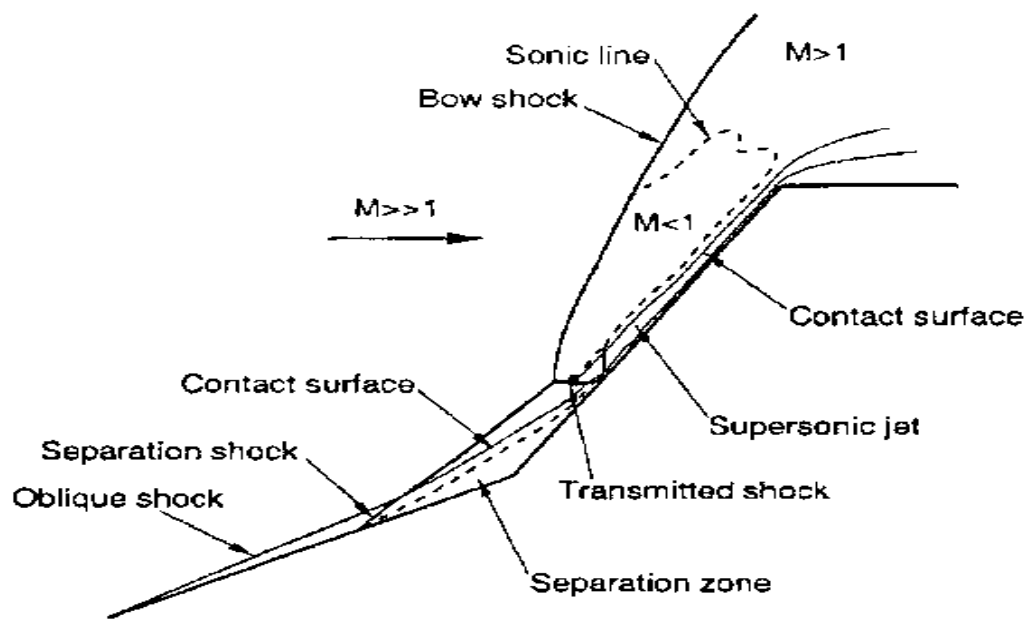

Figure 4.3:- Structure of Flow Field.

Figure 6.3 shows the diagram of the flow field structure. The first cone with relatively small angle compresses the flow and generates the attached shock wave. The Mach number decreses after the first shock wave, but the flow is stil supersonic. The second shock wave is formed after the flow compression by the second cone. Adverse pressure gradient is formed in the second shock wave, inducing the boundary layer flow's seperation at the first cone, finally forming the seperation shock wave.

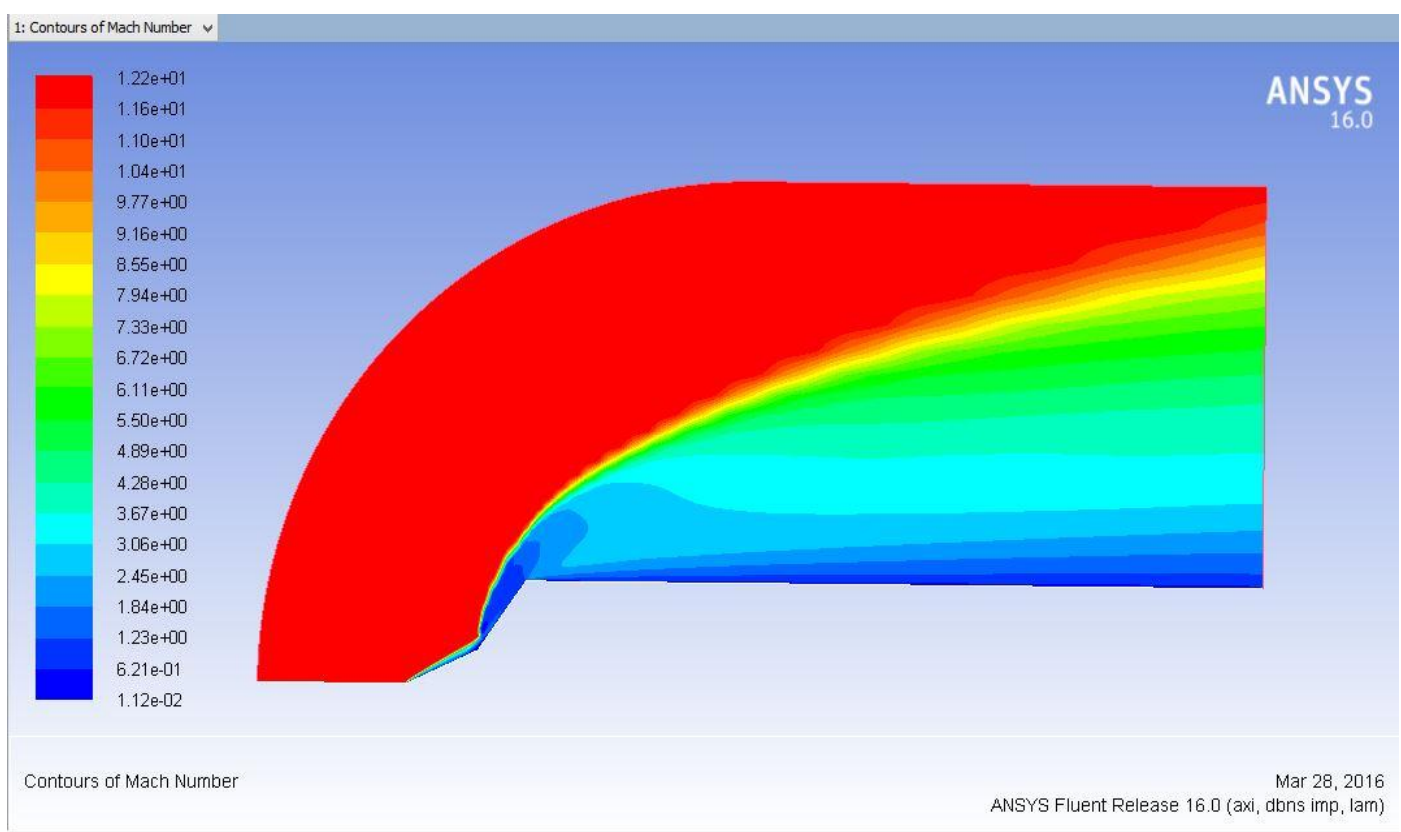

Figure 4.4:- Mach number palettes on $25-55^{\circ}$ Double Cone Model 


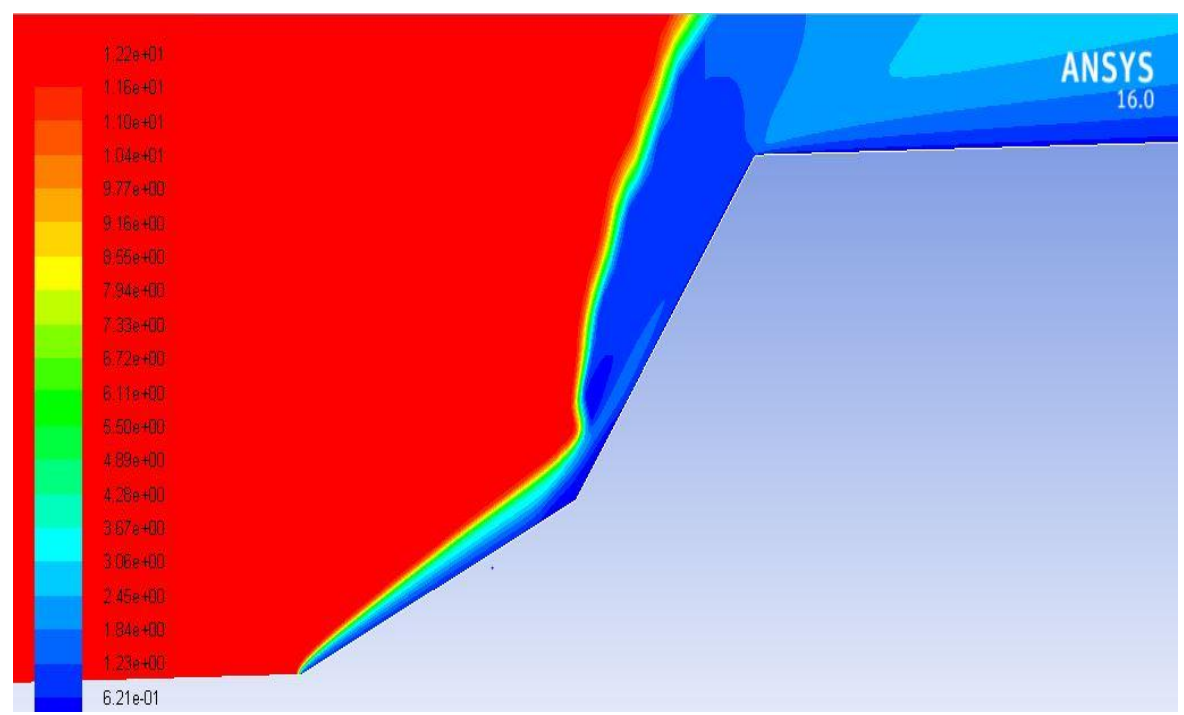

Figure 4.5:- Flow Features zoomed on the Mach Number Palette.

The flow field around the double cone configuration is illustrated on the basis of Mach number contours in fig 4.4. The flow seperates at the intersection between both cones. Infront of the seperation bubble a weak shock wave is formed which interacts with the weak shock from the tio of the first cone. Further downstream the shock wave interacts with the strong bow shock in front of the second cone forming a complex shock-shock interaction.

The attached leading edge shock wave interacts with a detached bow shock wave formed from the second cone, and this interaction produces a transmitted shock wave that impinges in the second cone surface. This produces very high surface pressures and heat transfer rates on the second cone. Because of the high pressures at the impingement location, the flow separates near the cone-cone juncture and a recirculation zone develops, which in turn alters the shock interaction. The size of the separation zone is very sensitive to the shock angles and to the strength of the shock interaction. Downstream of the shock impingement location, a supersonic jet develops near the second cone surface.

Most of the flow features could be captured in the simulation like the oblique shock, bow shock, sonic line, and supersonic jet.

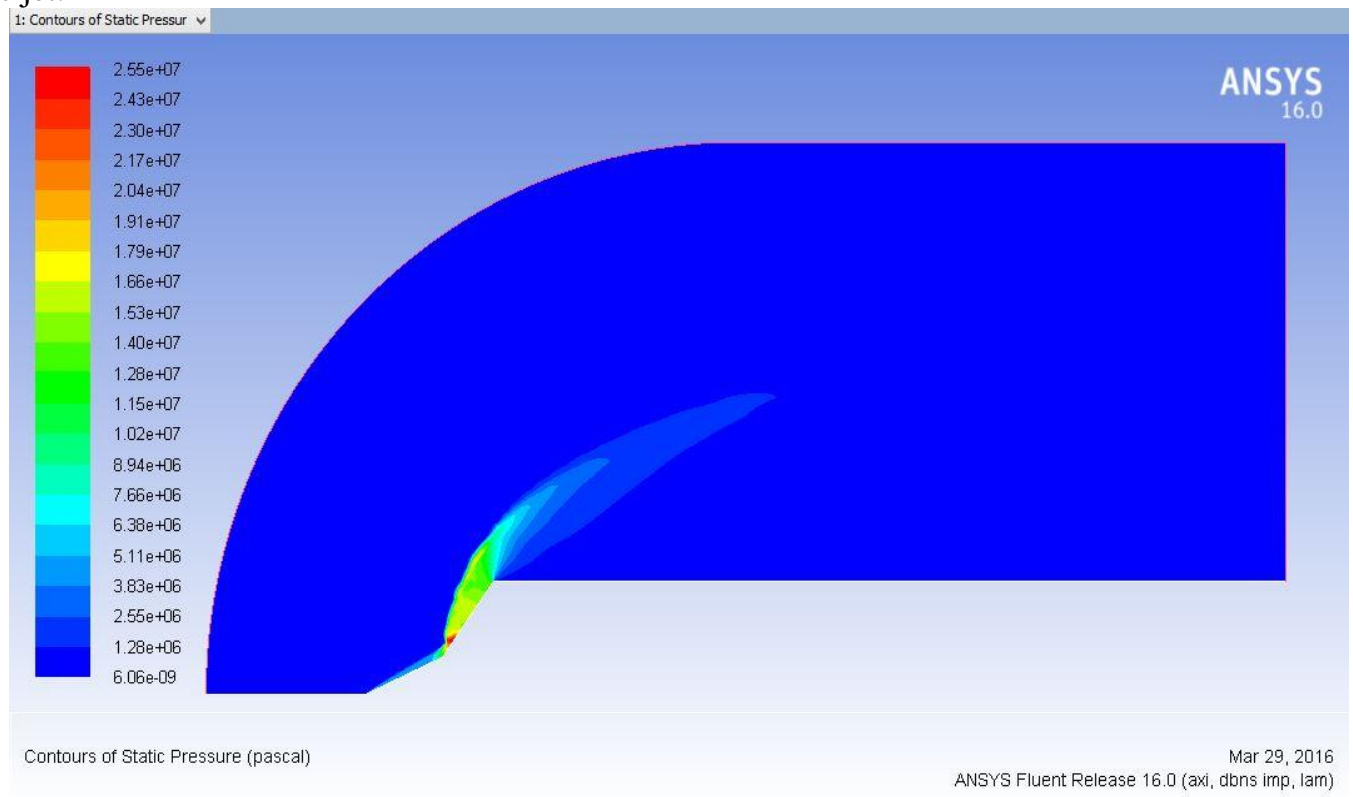

Figure 4.6:- Static Pressure palette on 25-55 Double cone Model. 


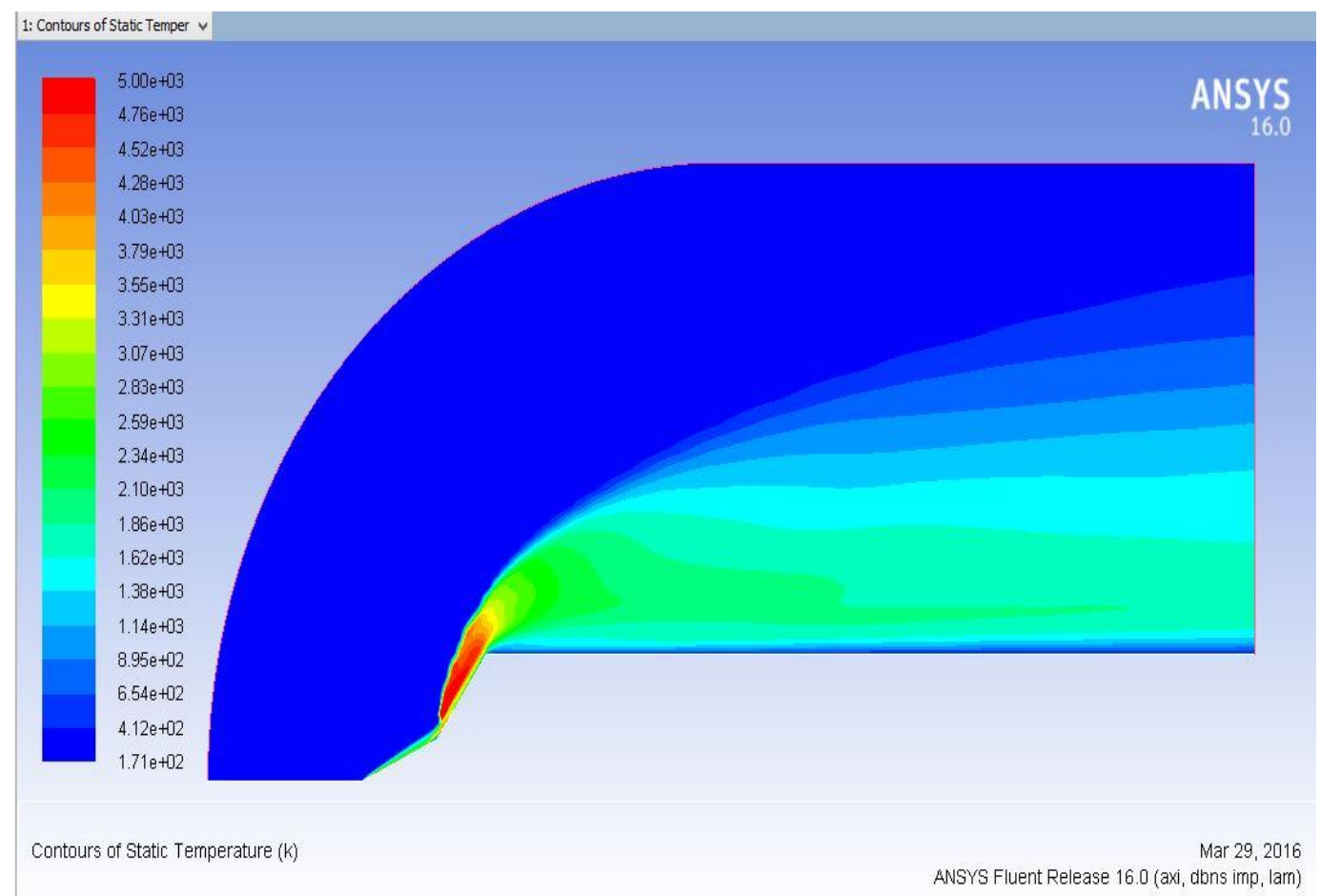

Figure 4.7:- Static Temperature palette on 25-55 Double cone Model.

The Mach number contour gives an idea about the flowfield over a typical sharp tipped $25-55^{\circ}$ Double cone configuration. The cone bow shock merges with the one produced by the flow deflection at separation to form a single strengthened wave. A shock wave or compression wave is produced by the outward bending of the shear layer as it reattaches on the downstream cone. Both of these waves converge downstream above the surface of the rear cone where they produce a complex and energetic interaction region that gives rise to sharp pressure and heat transfer peak on the surface.

\section{Conclusion and Future Enhancement:-}

\section{Summary:-}

In this project, an attempt has been made to study the flow field structure on a double cone model of $25 / 55^{\circ}$ half angles and to measure the surface heat flux and pressure using ANSYS Fluent. For the study, the experimental values are taken from the database of CUBRC to confirm the accuracy of the numerical simulation software for laminar flow hypersonic separation flow.

The test cases chosen for this work constituted a very significant challenge to the CFD community and it is impressive to see the results compared with the experimental data. The body shape induces complex shock interaction phenomena and separation, which makes these flows extremely challenging to calculate.

\section{Conclusion:-}

Through numerical simulation and analysis of the sharp double cone configuration, the following conclusions are arrived:

- There has been an exceptionally good similarity between the experimental values obtained in the LENS facility at Calspan University at Buffalo Research centre and the obtained numerical results using evaluation version of Fluent Software.

- Numerical Simulations are carried out to understand the flow features over the double cone model at various freestream conditions.

- The prediction shows the typical shock patterns over the geometry and compared well with the experimental values. 
- The variation in the experimental and computational plots can be because the shock angles in this flow are highly sensitive to the chemical composition of the flow, this will result in significant changes in the pressure and heat transfer layer distribution as the composition of the gas in the freestream and in the shock over the body is changed.

- Consistency is observed between the surface pressure and heat flux- separation and peak reattachment levels occur at about the same location within each run.

\section{References:-}

1. Li Jing,Xiao Hong and $\mathrm{Wu}$ Di., "Simulation and experimental validation of Hypersonic shock wave interaction”, Journal of Applied Sciences, Engineering and Technology, vol 22, 2040-7459 (2013)

2. B. Vasudevan, S.P.Srikanth, H.U. Shashidhar, G.Jagadeesh., "Surface pressure and heat transfer measurements in the unsteady separated hypersonic flow field over double cones", Proceedings of the $24^{\text {th }}$ International Symposium on Shock waves Beijing, China July 11-16,2004, pp 389-394 (2004)

3. Marie-Claude Druguet, Graham V. Candler and Ioannis Nompelis., "Effect of Numerics on Navier-stokes computations of Hypersonic Double-Cone Flows", AIAA Journal, vol 43(2005)

4. Matthew MacLean, Michael S.Holden, Aaron Dufrene., "Measurements of Real Gas Effects on Regions of Laminar Shock Wave/Boundary Layer Interaction in Hypervelocity Flows", AIAA Aviation 2014 (2014)

5. Peter A. Gnoffo., "CFD Validation Studies for Hypersonic Flow Prediction", AIAA Journal (2001)

6. Michael S.Holden, Timothy P.Wadhams and Matthew MacLean., "A Review of Experimental Studies with the Double Cone and Hollow Cylinder/Flare Configurations in the LENS Hypervelocity Tunnels and comparisons with Navier-Stokes and DSMC Computations", AIAA Journal, 2010-1281 (2010)

7. Michael Holden., "Experimental Studies of Laminar Seperated Flows Induced by Shock Wave/Boundary Layer and Shock/Shock Interaction in Hypersonic Flows for CFD Validation", AIAA Journal, 2000-0930 (2000)

8. Anderson, John D., Jr.: Computational Fluid Dynamics: The basics with applications, Indian Edition.

9. Anderson, John D., Jr.: Hypersonic and High Temperature Gas Dynamics, McGraw-Hill, New York, 1989.

10. Anderson, John D., Jr.: Fundamentals of Aerodynamics, 2d ed., McGraw-Hill, New York, 1991.

11. J. Blazek: Computational Fluid Dynamics: Principles and Applications, Elsevier 2001.

12. John F. Wendt Editor: Computational Fluid Dynamics- An Introduction, 3rd Edition.

13. ANSYS Modeling and Meshing Guide: ANSYS Release 10.0 August 2005

14. Ya. B. Zel'dovich: Theory Of Shock Waves and Introduction to Gas Dynamics: Foreign Technology Division; 1967

15. Patankar, S. V.: Numerical Heat Transfer and Fluid Flow, Hemisphere, New York, 1980.

16. Rubbert, Paul, and Dockan Kwak(eds): AIAA 10th Computational Fluid Dynamics Conference, June 2427,1991.

17. Anderson, John D., Jr.: Modern Compressible Flow: With Historical Perspective,2d ed.' McGraw-Hill, New York, 1990

18. Schlichting, H.: Boundary Layer Theory, 7th ed., McGraw-Hill, New York, 1979.

19. Anderson, Dale A., John C. Tannehill, and Richard H. Pletcher: Computational Fluid Mechanics and Heat Transfer, McGraw-Hill, New York, 1984. 